
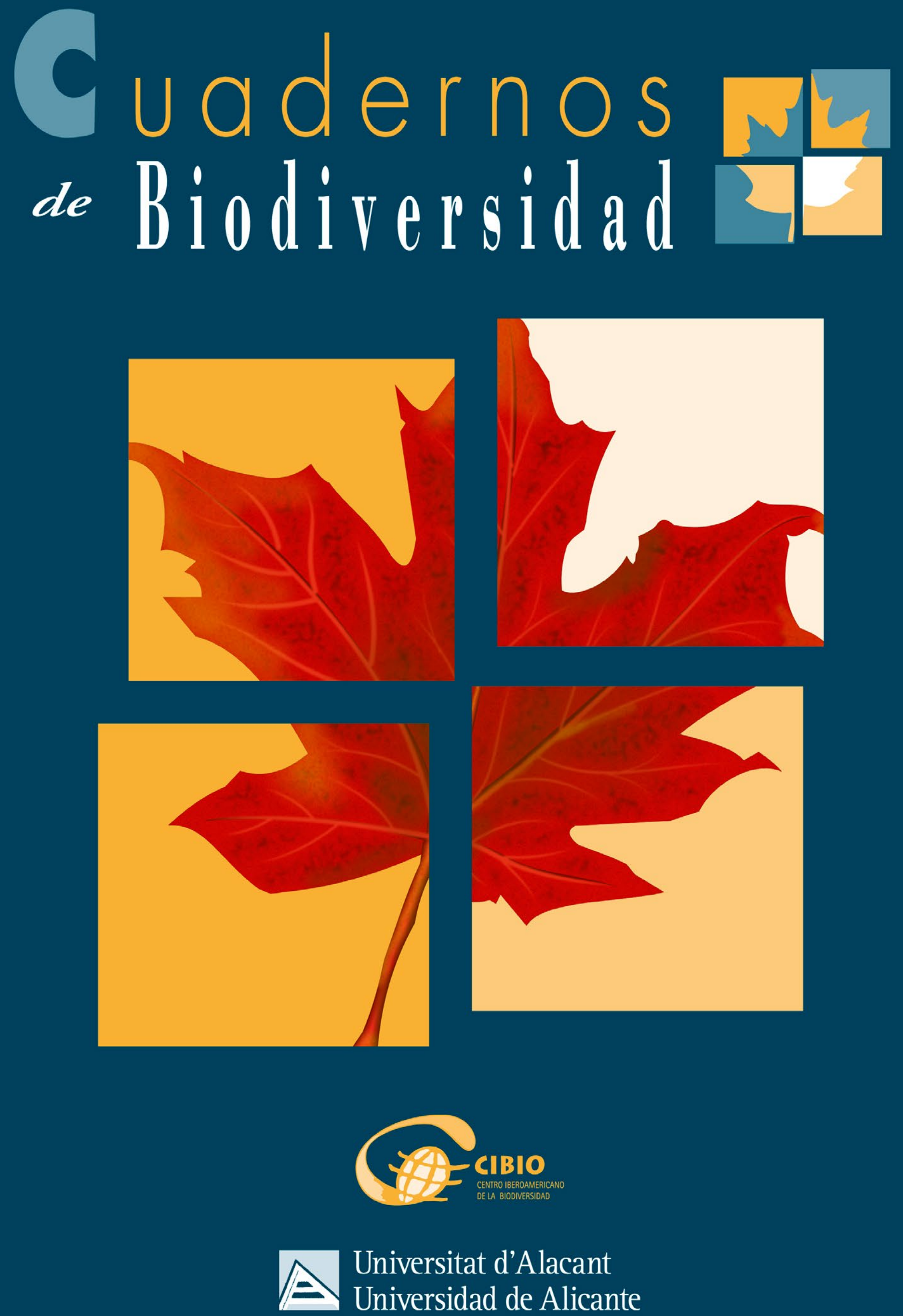


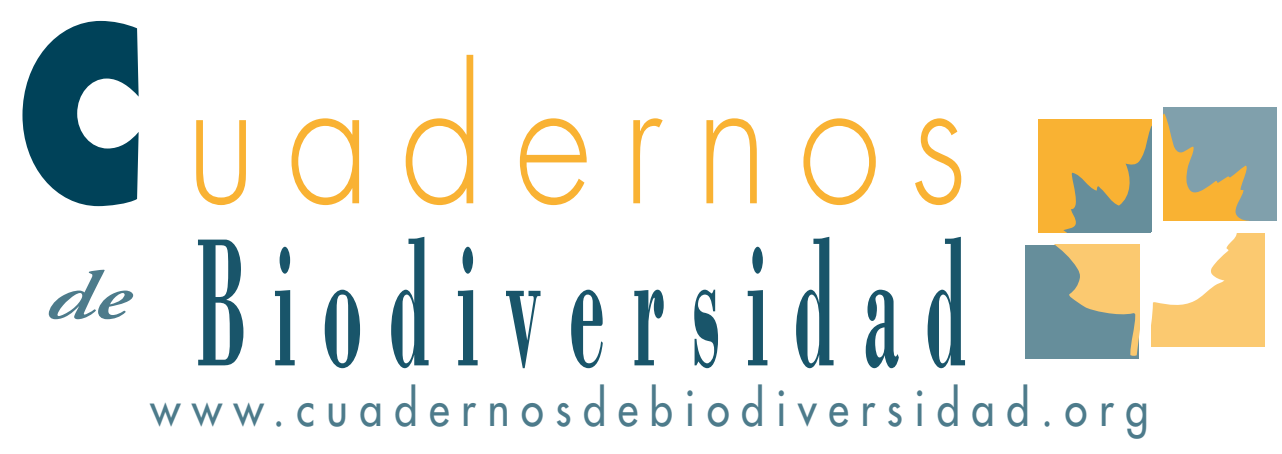

\section{Coleópteros saproxílicos en bosques mediterráneos de Chile: primeros pasos hacia su conocimiento y conservación}

\section{Alejandra García-López ${ }^{1}$}

i Laboratorio de Ecología de Ambientes Fragmentados, Universidad de Chile. Av. Santa Rosa i i735, La Pintana, Santiago, Chile

\section{ABSTRACT}

Mediterranean ecosystems have been recognized as a priority for biodiversity conservation due to their high levels of species richness and endemism. In South America, these environments are restricted to central Chile and represent a biodiversity hotspot. The study of saproxylic beetles in this area is an unexplored topic, despite the ecological role they play in the ecosystems and their potential usefulness for monitoring the forests conservation degree. The present study highlights the importance of the saproxylic beetles diversity in Mediterranean forests of Chile. These ecosystems are characterized by a high heterogeneity of vegetations and by the presence of fragmented nearly zones that surround the protected areas and show different perturbation degrees. We also enhance the importance of the preservation of old native trees in those fragmented areas that can help sustain the biodiversity and have a significant conservation value for saproxylic fauna.
Key words: Diversity turnover, indicator species, saproxylic beetles, species distribution, Reserva Nacional Río Clarillo

\section{PAPEL DE LOS COLEÓPTEROS SAPROXÍLICOS EN LOS ECOSISTEMAS}

La dinámica funcional de los ecosistemas se encuentra regida por procesos ecológicos de los que derivan importantes servicios ecosistémicos (Escobar \& Maas, 2008; Díaz et al., 2006; Martín-López et al., 2007). Entre estos procesos ecológicos, la descomposición de la materia orgánica resulta fundamental ya que contribuye al reciclaje de nutrientes para formación y fertilidad del suelo (Chapin et al., 2002, Escobar \& Maass 2008). Uno de los principales almacenes de nutrientes se encuentra en la madera muerta presente en zonas forestales, la cual se ha convertido en un importante tema de investigación en las últimas décadas (p. ej. Laiho $\&$ 
Prescott, 1999; Harmon et al., 1986; van Geffen et al., 2010) puesto que a través de su descomposición dichos nutrientes son gradualmente liberados al ecosistema donde además constituye el hábitat de un gran porcentaje de organismos (Speight, 1989; Grove, 2002).

La descomposición de la madera en los bosques está mediada por la acción de los organismos saproxílicos. Estos organismos son aquellos que, en al menos una parte de su ciclo biológico, dependen de la madera muerta o en proceso de descomposición, de los hongos que colonizan dicha madera, o bien se encuentran asociados a la presencia de otras especies estrictamente saproxílicas (Alexander, 2008; Speight, 1989). A pesar del rol ecológico crucial que desempeñan, contribuyendo a la producción primaria mediante el suministro de nutrientes a las plantas (Dajoz, 1998; Didham et al., 1996; Schlaghamersky, 2003) y participando en la descomposición y el reciclaje de materia vegetal (Davies et al. 2008; Micó et al., 2011), los saproxílicos son uno de los grupos de organismos más amenazados actualmente debido a la degradación de los ecosistemas forestales (Alexander, 2004; Jonsell et al., 1998; Dajoz, 2000; Haslett, 2007; Nieto \& Alexander 2010; Siitonen, 2001; Speight, 1989).

En las últimas décadas los coleópteros saproxílicos han sido sujeto de numerosas investigaciones, principalmente en Europa, en las que se ha puesto de manifiesto la excepcional riqueza y diversidad de especies del grupo, la importancia de su función en los ecosistemas, su utilidad como indicador de características forestales y el importante impacto negativo que la pérdida y perturbación de áreas forestales tiene sobre sus comunidades (ver Buse et al., 2010; Lachat et al., 2012; Ranius \& Jansson 2000; Sebek et al., 2012; Stokland et al., 2012). Asimismo, el aumento del interés en la investigación de esta fauna ha producido un creciente número de trabajos sobre los factores que influyen en su diversidad. Así, distintos estudios subrayan la influencia de diversas variables ambientales sobre las comunidades de coleópteros saproxílicos como pueden ser el volumen y diversidad de la madera muerta, el grado de apertura del bosque y exposición solar ligadas a la temperatura y humedad, la presencia de oquedades en los árboles y la especie arbórea dominante (Grove, 2002;
Jonsell et al., 2007; Lindhe et al., 2005; Müller et al., 2010; Ranius, 2002; Ranius \& Jansson, 2000; Sverdrup-Thygeson et al., 2010). Los ensambles de coleópteros saproxílicos y su composición de especies y grupos funcionales también se han visto afectados por el grado de perturbación del bosque (Buse et al., 2010) y se ha observado la influencia de la escala de estudio en la que la heterogeneidad del paisaje y los distintos tipos de bosque que la conforman aumentan la riqueza y diversidad del grupo (Bergman et al., 2012; Müller \& Goßner, 2010; Saint-Germain et al., 2006; Ulyshen \& Hanula, 2009). Este efecto positivo de la heterogeneidad espacial sobre la diversidad muestra una especial importancia en bosques mediterráneos. En estos ecosistemas la fauna de coleópteros saproxílicos ha mostrado un alto grado de diversidad y singularidad (Quinto et al., 2012, 2013; Ricarte et al., 2009; Ramírez-Hernández et al., 2014) relacionado con la heterogeneidad espacial de la vegetación constituida por diferentes tipos de bosque y zonas de matorral y pastizal que crean un auténtico mosaico de vegetación (Micó et al., 2013). En estas zonas, análisis de partición de diversidad han demostrado que el recambio de especies entre diferentes tipos de bosque (diversidad beta) presenta valores muy elevados y contribuye a la diversidad total (diversidad gamma) en mayor medida que la diversidad alfa (riqueza local de especies) (Micó et al., 2013; Ricarte et al., 2011).

\section{IMPORTANCIA DE LOS ECOSISTEMAS MEDITERRÁNEOS. EL CASO DE CHILE}

Las regiones mediterráneas del mundo son conocidas por albergar niveles especialmente altos de riqueza de especies y endemismos (Cowling et al., 1996; Heywood 1993). Sin embargo, hoy en día los ecosistemas pertenecientes a estas áreas mediterráneas se han convertido en los más amenazados del planeta dada la larga historia de actividades humanas desarrolladas en estas zonas (Armesto et al., 2007; Naveh and Dan, 1973; Ramírez-Hernández et al., 2014; Underwood et al., 2009), razón por la cual estas áreas han sido reconocidas como prioritarias en materia de conservación de la biodiversidad (Myers et al., 2000; Sala et al., 2000). Los ambientes medi- 
terráneos de Sudamérica se encuentran confinados a una estrecha banda de unos mil kilómetros de longitud en la zona centro-norte de Chile $\left(30-36^{\circ}\right.$ S) (Arroyo et al., 1995, 1999). Varios estudios en esta región han subrayado el alto nivel de aislamiento geográfico que la caracteriza, así como los elevados niveles de diversidad biológica y endemicidad que presenta (Arroyo et al., 1995, 1999; Arroyo \& Cavieres 1997; Villagrán 1995), siendo considerada un importante hotspot de biodiversidad a nivel mundial (Arroyo et al., 1999; Myers 1990; Myers et al., 2000). Dada la reducida área de la región mediterránea chilena, los impactos de la actividad antrópica han resultado especialmente serios en sus ecosistemas (Armesto et al., 2007; Arroyo et al., 1995, 1999; Donoso \& Lara 1995). La conservación de la diversidad asociada a ellos resulta un reto extremadamente importante teniendo en cuenta que menos del $1 \%$ de las áreas actualmente protegidas en Chile se encuentran localizadas dentro de la región mediterránea del país (Sierralta et al., 2011).

A pesar de su función clave en los ecosistemas forestales y del creciente desarrollo de su investigación en otros países, hoy en día el estudio de la fauna de coleópteros saproxílicos en Chile, su diversidad y funcionalidad, es un tema prácticamente inexplo- rado. Algunos autores han puesto de manifiesto la importancia de la madera muerta y de su descomposición en el mantenimiento de los ecosistemas forestales chilenos (Carmona et al., 2002; Schlegel \& Donoso 2008), sin embargo, los coleópteros saproxílicos han recibido muy poca atención y, en este sentido, los bosques mediterráneos del país no son una excepción.

La Reserva Nacional de Río Clarillo constituye un ejemplo de estos bosques mediterráneos (Fig. 1). Esta área protegida creada por el Ministerio de Agricultura en el año 1982 (CONAF, 1996), está situada en la región central de Chile, a $45 \mathrm{~km}$ al SO de Santiago $\left(33^{\circ} 46^{\prime} 00^{\prime \prime S}, 70^{\circ} 27^{\prime} 00^{\prime \prime O}\right)$. Cubre una superficie de 13085 ha (Niemeyer et al., 2002) e incluye un importante área de matorral y bosques esclerófilos considerados una de las formaciones vegetales características de las áreas mediterráneas (Gajardo, 1994; Quezel, 2004) (Fig. 2, 3). La Reserva presenta un clima típicamente mediterráneo con una marcada oscilación estacional, tanto en precipitaciones como en temperaturas mensuales medias (Niemeyer et al., 2002). Adyacentes a la Reserva se encuentran áreas fuertemente influenciadas por actividades humanas como la agricultura, la ganadería, la presencia de caminos y áreas urbanas.

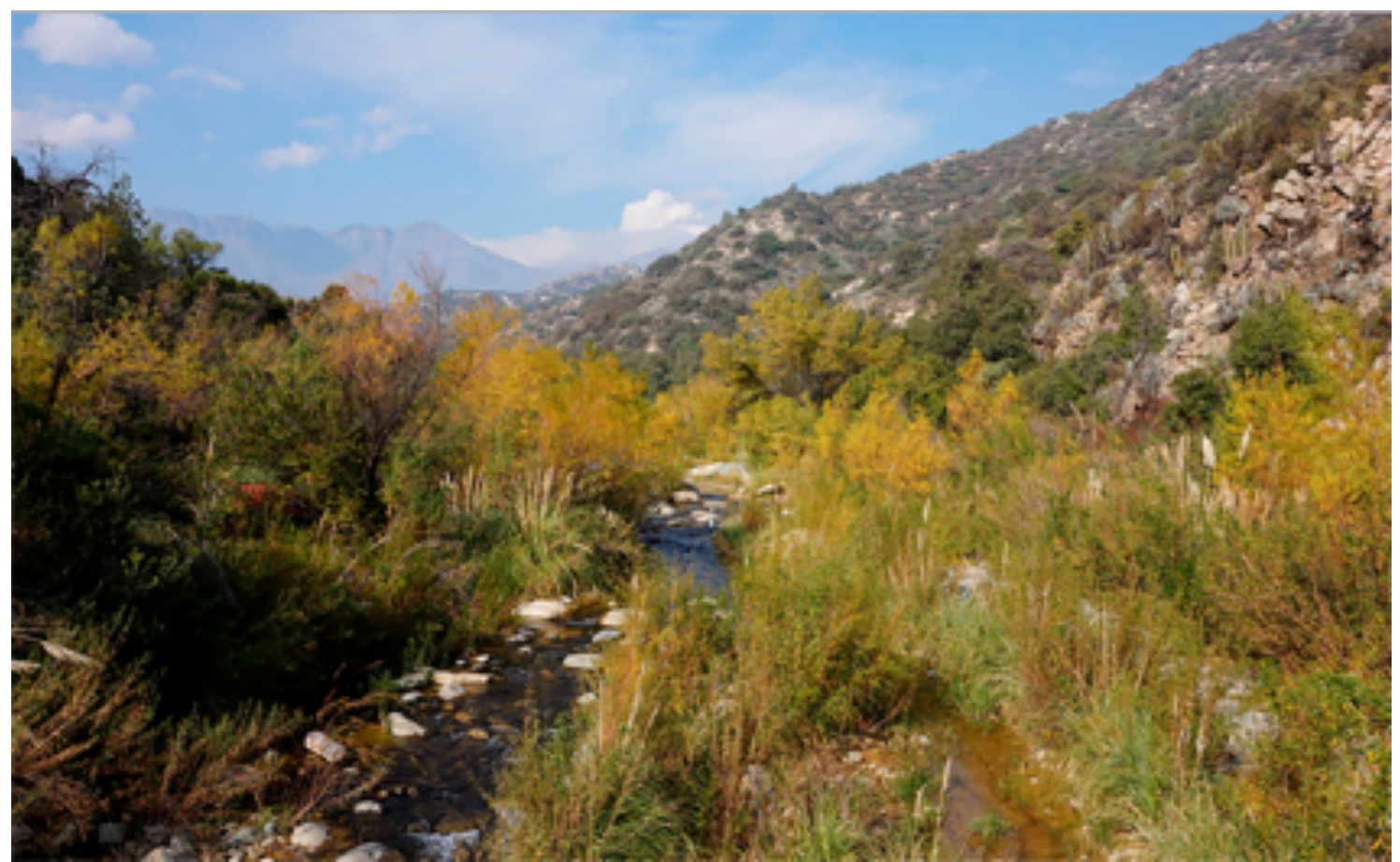

Figura 1. Reserva Nacional del Río Clarillo, Chile. 




Figura 2. Localización geográfica de la Reserva Nacional del Río Clarillo en la zona mediterránea de Chile e imagen de satélite de la zona.

Es en esta zona de Chile donde a día de hoy se han llevado a cabo los primeros análisis sobre la diversidad de coleópteros saproxílicos en los bosques mediterráneos de Chile (García-López et al., 2016), que aportan los primeros datos sobre las variables que influyen en la distribución de las especies y el impacto de la perturbación antrópica sobre sus ensambles.
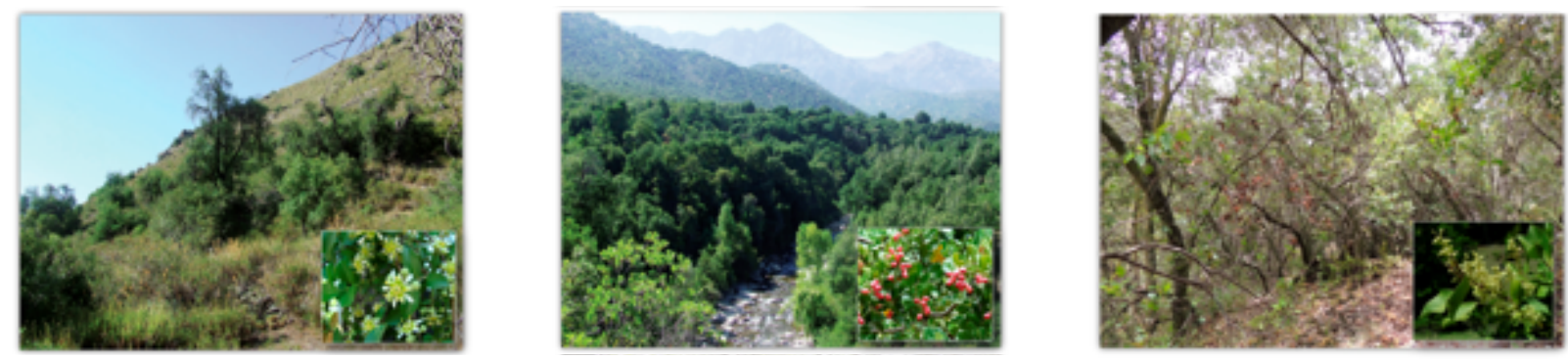

Figura 3. Algunos tipos de bosque mediterráneo presentes en la Reserva. De izquiera a derecha: Bosque de Quillay y detalle de la flor de Quillaja saponaria, bosque de Peumo y detalle del fruto de Cryptocarya alba, bosque de Radal y detalle de la flor de Lomatia hirsuta.

Durante el mencionado estudio se recolectaron e identificaron a nivel de especie un total de 156 especies y 3083 individuos pertenecientes a 34 familias de coleópteros saproxílicos, siendo Elateridae y Ptinidae las familias que mostraron una mayor representación en cuanto al número de especies (Tabla 1). Algunos de los individuos colectados pertenecen a endemismos de Chile (Solervicens 2014), como la especie xilófaga Candanius gracillimus (Candeze 1889) (Elateridae), la saprófaga Trogoderma rubiginosum (Solier, 1849) (Dermestidae) y las especies pertenecientes al género Allobregmus Español, 1970 (Ptinidae). 
En general, los autores encontraron diferencias significativas de diversidad tanto entre tipos de bosque como entre estaciones, poniendo de relevancia el importante papel que, como se ha visto en otras áreas mediterráneas (Micó et al., 2013; RamírezHernández et al., 2014), presenta la variable heterogeneidad espacio-temporal de estas zonas sobre la diversidad que albergan.

Resulta especialmente interesante el hecho de que mayores valores de riqueza están siempre asociados a variables ligadas a la edad y el tamaño del árbol. Este dato subraya la importancia que la presencia de árboles viejos y de gran tamaño tiene para la biodiversidad saproxílica y el papel clave que presentan en la conservación de esta fauna (Lindhe et al., 2005; Stokland et al., 2012). En las zonas perturbadas que se encuentran adyacentes a las áreas protegidas suelen aparecer árboles grandes y añosos que han permanecido sin talar a lo largo del tiempo de cara a mantenerlos como ornamentales o para el aprovechamiento de su sombra. Estos árboles actúan como refugios para las especies saproxílicas que encuentran en ellos los microhábitats adecuados donde desarrollarse.

\section{CONCLUSIONES}

A pesar de la baja representación de los ecosistemas mediterráneos dentro de las áreas protegidas de Chile (menos del 1\%) (Pliscoff \& Fuentes-Castillo, 2011; Sierralta et al., 2011) y del grado de perturbación que estos ecosistemas sufren en el país debido a los impactos de la actividad antrópica (Armesto et al., 2007), se ha comprobado que estas las áreas mediterráneas chilenas albergan ambientes saproxílicos muy diversos que promueven la diversidad de especies de coleópteros saproxílicos.

Los primeros resultados sobre esta fauna en el país ponen de relevancia que en los programas de conservación de los bosques mediterráneos chilenos no sólo se debe prestar atención a las áreas protegidas, sino también a otras zonas cercanas fragmentadas, donde acciones sencillas como la conservación de
Tabla 1. Riqueza de especies y número de individuos recolectados.

\begin{tabular}{|c|c|c|}
\hline Familia & $\begin{array}{c}\text { No de } \\
\text { especies }\end{array}$ & $\begin{array}{c}\text { No de } \\
\text { individuos }\end{array}$ \\
\hline Aderidae & 1 & 26 \\
\hline Anthicidae & 3 & 52 \\
\hline Anthribidae & 1 & 1 \\
\hline Archaeocryticidae & 1 & 20 \\
\hline Bostrichidae & 5 & 42 \\
\hline Buprestidae & 6 & 29 \\
\hline Cantharidae & 4 & 91 \\
\hline Carabidae & 11 & 421 \\
\hline Cavognathidae & 1 & 5 \\
\hline Cerambycidae & 9 & 30 \\
\hline Ciidae & 1 & 2 \\
\hline Cleridae & 9 & 70 \\
\hline Corylophidae & 1 & 2 \\
\hline Cryptophagidae & 5 & 120 \\
\hline Cupedidae & 1 & 1 \\
\hline Curculionidae & 11 & 97 \\
\hline Dermestidae & 7 & 253 \\
\hline Elateridae & 16 & 135 \\
\hline Histeridae & 2 & 2 \\
\hline Lampyridae & 1 & 4 \\
\hline Latridiidae & 1 & 25 \\
\hline Leiodidae & 2 & 3 \\
\hline Melyridae & 10 & 695 \\
\hline Mordellidae & 4 & 8 \\
\hline Mycetophagidae & 1 & 15 \\
\hline Nitidulidae & 3 & 13 \\
\hline Ptiliidae & 1 & 2 \\
\hline Ptinidae & 22 & 539 \\
\hline Scarabaeidae & 6 & 104 \\
\hline Scirtidae & 3 & 22 \\
\hline Scraptiidae & 1 & 240 \\
\hline Silvanidae & 1 & 1 \\
\hline Tenebrionidae & 4 & 12 \\
\hline Trogossitidae & 1 & 1 \\
\hline
\end{tabular}


árboles maduros nativos pueden conseguir que áreas perturbadas contengan un elevado número de especies saproxílicas y puedan actuar como sitios clave en el mantenimiento de la biodiversidad no solo en esta región, sino también en otros ecosistemas alrededor del mundo.

Hoy en día la información existente sobre la fauna saproxílica en Chile es muy escasa. Esperamos que los primeros datos que comienzan a aparecer sobre estas especies y que ponen de relevancia su enorme diversidad e importancia, sirvan de base $y$ herramienta para futuros estudios que nos ayuden a conocer y entender la distribución de estas especies y a ser eficaces en el desarrollo de estrategias para su conservación.

\section{AGRADECIMIENTOS}

Financiación a cargo del proyecto FONDECYT 3140322 (Fondo Nacional de Ciencia y Tecnología), Gobierno de Chile.

\section{REFERENCIAS}

Alexander, K.N.A. 2004. Revision of the index of ecological continuity as used for saproxylic beetles. English Nature Research Report 574. English Nature, Peterborough.

Alexander, K.N.A. 2008. Tree biology and saproxylic Coleoptera: Issues of definitions and conservation language. Rev. Ecol-Terre Vie. 63:1-5.

Arroyo, M.T.K. \& Cavieres, L. 1997. The Mediterraneantype climate flora of central Chile - What do we know and how can we assure its protection? Noticiero de Biología (Chile) 5:48-56.

Armesto, J.J., Arroyo, M.T.K. \& Hinojosa, L.F. 2007. The Mediterranean environment. En: Veblen T.T., Young K.R. \& Orme A.R. (eds.), The physical geography of South America. Oxford University Press. pp. 184-199.

Arroyo, M.T.K, Cavieres, L., Marticorena, C. \& MuñozSchick, M. 1995. Convergence in the Mediterranean floras in central Chile and California: Insights from comparative biogeography. En: Arroyo, M.T.K., Zedler, P.H. \& Fox, M.D. (eds.), Ecology and biogeography of Mediterranean ecosystems in Chile, California, and Australia. Springer-Verlag, New York. pp. 43-88.
Arroyo, M.T.K., Rozzi, R., Simonetti, J.A. \& Salaberry, M. 1999. Central Chile. En: Mittermeier, R.A., Myers, N. \& Mittermeier, C.G. (eds.), Hotspots. Earth's biologically richest and most endangered terrestrial ecoregions. CEMEX, Mexico. pp. 161-171.

Bergman, K.O., Jansson, N., Claesson, K., Palmer, M.W. \& Milberg, P. 2012. How much and at what scale? Multiscale analyses as decision support for conservation of saproxylic oak beetles. Forest Ecol. Manag. 265:133141.

Bouget, C., Brustel, H., Brin, A. \& Noblecourt, T. 2008. Sampling saproxylic beetles with window flight traps: methodological insights. Rev. Ecol-Terre. Vie. 63:13-24.

Buse, J., Levanony, T., Timm, A., Dayan, T. \& Assmann, T. 2010. Saproxylic beetle assemblages in the Mediterranean region: Impact of forest management on richness and structure. Forest Ecol. Manag. 259:13761384. doi:10.1016/j.foreco.2010.01.004.

Carmona, M.R., Armesto, J.J., Aravena, J.C. \& Pérez, C.A. 2002. Coarse woody debris biomass in successional and primary temperate forests in Chiloé Island, Chile. Forest Ecol. Manag. 164:265-275. doi:10.1016/S03781127(01)00602-8

Chapin, I.F.S., Matson, P.A. \& Mooney, H.A. 2002. Principles of terrestrial ecosystem ecology. Springer, Nueva York. pp. 529.

CONAF, Corporación Nacional Forestal. 1996. Plan de manejo Reserva Nacional Río Clarillo. Documento de Trabajo No. 247, Unidad de gestión Patrimonio Silvestre, Corporación Nacional Forestal, Región Metropolitana, Santiago, Chile.

Cowling, R., Rundel, P., Lamont, B., Arroyo, M. \& Arianoutsou, M. 1996. Plant diversity in Mediterranean climate regions. Trends. Ecol. Evol. 11:362-366.

Dajoz, R. 1998. Les insectes et la forêt: Rôle et diversité des insectes dans le milieu forestier. Lavoisier. Technique and Documentation, Paris. pp. 594.

Dajoz, R. 2000. Insects and forests: the role and diversity of insects in the forest environment. Intercept, Andover. pp. 668.

Davies, Z.G., Tyler, C., Stewart, G.B. \& Pullin, A.S. 2008. Are current management recommendations for saproxylic invertebrates effective? A systematic review. Biodiv. Conserv. 17:209-234. 
Díaz, S., Fargione, J.F., Chapin, S., \& Tilman, D. 2006. Biodiversity Loss Threatens Human Well-Being. PLoS. Biol. 4:e277.

Didham, R.K., Ghazoul, J., Stork, N.E. \& Davis, A.J. 1996. Insects in fragmented forests: a functional approach. Trends Ecol. Evol. 11:255-260.

Donoso, C., Lara, A. 1995. Utilización de los bosques nativos en Chile: pasado, presente y futuro. En: Armesto, J.J., Villagrán, C. \& Arroyo, M.T.K. (eds.), Ecología de los bosques nativos de Chile. Editorial Universitaria, Universidad de Chile, Santiago. pp 23-28.

Escobar, E. \& Maass, M. 2008. Capital natural de México, vol. I: Conocimiento actual de la biodiversidad. Conabio, México.

Gajardo, R. 1994. La Vegetación Natural de Chile. Clasificación y distribución geográfica. Editorial Universitaria, Santiago, Chile. pp. 165.

García-López, A., Martínez-Falcón, A.P., Micó, E., Estrada, P., Grez, A.A. 2016. Diversity distribution of saproxylic beetles in Chilean Mediterranean forest: influence of spatiotemporal heterogeneity and perturbation. J Insect Coserv, DOI 10.1007/s10841-016-9905-7.

Grove, S.J.2002. Saproxylicinsect ecology and the sustainable management of forests. Annu. Rev. Ecol. Syst. 33:1-23. doi:10.1146/annurev.ecolsys.33.010802.150507.

Harmon, M. E., Franklin, J.F., Swanson, F.J., Sollins, P.S., Gregory, V., Lattin, J.D., Anderson, N.H., Cline, S.P., Aumen, N.G., Sedell, J.R., Lienkaemper, G.W., Cromack, K.J. \& Cummins, K.W. 1986. Ecology of coarse woody debris in temperate ecosystems. En: MacFadyen, A. \& Ford, E.D. (eds.) Advances in ecological research. Orlando, FL: Academic Press, Inc.: 15: 133-302.

Heywood, H. 1993. Mediterranean floras and their significant in relation to world biodiversity. Dissertation. Parc Naturel Régional de la Corse and Conservatoire Botanique National de Porquerolles, France.

Jonsell, M., Hansson, J. \& Wedmo, L. 2007. Diversity of saproxylic beetle species in logging residues in Sweden: comparisons between tree species and diameters. Biol. Cons. 138:89-99.

Jonsell, M., Weslien, J. \& Ehnström, B. 1998. Substrate requirements of red-listed saproxylic invertebrates in Sweden. Biodivers. Conserv. 7:749-764.
Lachat, T., Wermelinger, B., Gossner, M.M., Bussler, H., Isacsson, G., Müller, J. 2012. Saproxylic beetles as indicator species for dead-wood amount and temperature in European beech forests. Ecol. Indic. 23:323-331. doi:10.1016/j.ecolind.2012.04.013.

Laiho, R. \& Prescott, C.E. 1999. The contribution of coarse woody debris to carbon, nitrogen, and phosphorus cycles in three Rocky Mountain coniferous forests. Can. J. Forest Res. 29:1592-1603.

Lindhe, A., Lindelöw, Å. \& Åsenblad, N. 2005. Saproxylic beetles in standing dead wood density in relation to substrate sun-exposure and diameter. Biodivers. Conserv. 14:3033-3053

Martín-López, B., González, J.A., Díaz, S., Castro, I. \& García-Llorente, M. 2007. Biodiversidad y bienestar humano: el papel de la diversidad funcional. Ecosistemas 16:69-80.

Micó, E., Juárez, M., Sánchez, A. \& Galante, E. 2011. Action of the saproxylic scarab larva Cetonia aurataeformis (Coleoptera: Scarabaeoidea: Cetoniidae) on woody substrates. J. Nat. Hist. 45:2527-2542.

Micó, E., García-López, A., Brustel, H., Padilla, A. \& Galante, E. 2013. Explaining the saproxylic beetle diversity of a protected Mediterranean area. Biodivers. Conserv. 22:889-904. doi:10.1007/s10531-013-0456-x.

Müller, J., Noss, R.F., Bussler, H. \& Brandl, R. 2010. Learning from a "benign neglect strategy" in a national park: Response of saproxylic beetles to dead wood accumulation. Biol. Conserv. 143:2559-2569. doi:10.1016/j.biocon.2010.06.024.

Müller, J. \& Goßner, M.M. 2010. Three-dimensional partitioning of diversity informs state-wide strategies for the conservation of saproxylic beetles. Biol. Conserv. 143:625-633. doi:10.1016/j.biocon.2009.11.027.

Myers, N., Mittermeier, R.A., Mittermeier, C.G., da Fonseca, G.A.B. \& Kent, J. 2000. Biodiversity hotspots for conservation priorities. Nature 403:853-858.

Naveh, Z. \& Dan, J. 1973. The human degradation of Mediterranean landscape in Israel. En: Di Castri, F., Monney, H. (eds.) Mediterranean type ecosystems. Springer, Berlin, pp. 373-390.

Niemeyer, H.M., Bustamante, R.O., Simonetti, J.A., Teillier, S., Fuentes-Contreras, E. \& Mella, J.E. 2002. Historia natural de la reserva nacional Río Clarillo: un espacio para aprender ecología. Impresos Socías, Santiago, Chile. 
Nieto, A. \& Alexander, K.N.A. 2010. European Red List of Saproxylic Beetles. Luxembourg: Publications Office of the European Union.

Pliscoff, P., Fuentes-Castillo, T. 2011. Representativeness of terrestrial ecosystems in Chile's protected area system. Environ. Conserv. 38:303-311. doi:10.1017/ S0376892911000208.

Quinto, J., Marcos-García, M.A., Díaz-Castelazo, C., RicoGray, V., Brustel, H., Galante, E. \& Micó, E. 2012. Breaking down Complex Saproxylic Communities: Understanding Sub-Networks Structure and Implications to Network Robustness. PLoS ONE. 7:1-9

Quinto, J., Marcos-García, M.A., Brustel, H., Galante, E. \& Micó, E. (2013) Effectiveness of three sampling methods to survey saproxylic beetle assemblages in Mediterranean woodland. J. Insect Conserv. 17:765776. doi:10.1007/s10841-013-9559-7.

Ramírez-Hernández, A., Micó, E., Marcos-García, M.A., Brustel, H. \& Galante, E. 2014. The "dehesa", a key ecosystem in maintaining the diversity of Mediterranean saproxylic insects (Coleoptera and Diptera: Syrphidae). Biodivers. Conserv. 23:2069-2086. doi:10.1007/s10531014-0705-7.

Ranius, T. \& Jansson, N. 2000. The influence of forest regrowth, original canopy cover and tree size on saproxylic beetles associated with old oaks. Biol. Conserv. 95:85-94.

Ranius, T. 2002. Influence of stand size and quality of tree hollows on saproxylic beetles in Sweden. Biol. Conserv. 103:85-91.

Ricarte, A., Jover, T.M., Marcos-García, A., Micó, E. \& Brustel, H. 2009. Saproxylic beetles (Coleoptera) and hoverflies (Diptera: Syrphidae) from a Mediterranean forest: towards a better understanding of their biology for species conservation. J. Nat. Hist. 43:583-607.

Ricarte, A., Marcos-García, M.A. \& Moreno, C.E. 2011. Assessing the effects of vegetation type on hoverfly (Diptera: Syrphidae) diversity in a Mediterranean landscape: implications for conservation. J. Insect Conserv. 15: 865-877.

Saint-Germain, M., Buddle, C.M., Drapeau, P. 2006. Sampling saproxylic Coleoptera: scale issues and the importance of behaviour. Environ. Entomol. 35:478487.
Sala, O.E., Chapin, F.S., Armesto, J.J., Berlow, E., Bloomfield, J., Dirzo, R., Huber-Sanwald, E., Huenneke, L.F., Jackson, R.B., Kinzig, A., Leemans, R., Lodge, D.M., Mooney, H.A., Oesterheld, M., Poff, N.L., Sykes, M.T., Walker, B.H., Walker, M. \& Wall, D.H. 2000. Global biodiversity scenarios for the year 2100. Science 287:1770-1774.

Schlaghamersky, D.J. 2003. Saproxylic invertebrate of floodplains, a particularly endangered component of biodiversity. En: Mason, F., Nardi, G. \& Tisato, M. (eds.) Dead wood: a key to biodiversity, Proceedings of the International Symposium 29-31 May 2003. Mantova (Italy): Compagnia delle Foreste. 99 p.

Schlegel, B.C. \& Donoso, P.J. 2008. Effects of forest type and stand structure on coarse woody debris in old-growth rainforests in the Valdivian Andes, southcentral Chile. Forest Ecol. Manag. 255:1906-1914. doi:10.1016/j.foreco.2007.12.013.

Sebek, P., Barnouin, T., Brin, A., Brustel, H., Dufrêne, M., Gosselin, F., Meriguet, B., Micas, L., Noblecourt, T., Rose, O., Velle, L. \& Bouget, C. 2012. A test for assessment of saproxylic beetle biodiversity using subsets of "monitoring species". Eco.l Indic. 20:304315. doi:10.1016/j.ecolind.2012.02.033.

Sierralta, L., Serrano, R., Rovira, J. \& Cortés, C. 2011. Las áreas protegidas de Chile. Antecedentes, institucionalidad, estadísticas y desafíos. Ministerio del Medio Ambiente. Chile.

Siitonen, J. 2001. Forest Management, Coarse Woody Debris and Saproxylic Organisms: Fennoscandian Boreal Forests as an Example. Ecol. Bull. 49:11-41.

Speight, M.C.D. 1989. Saproxylic invertebrates and their conservation. Nature and environment, series 42. Council of Europe, Strasbourg, France.

Stokland, J., Siitonen, J. \& Jonsson, B. 2012. Biodiversity in dead wood. Cambridge University Press. Cambridge. pp. 559.

Sverdrup-Thygeson, A., Skarpaas, O. \& Ødegaard, F. 2010. Hollow oaks and beetle conservation: the significance of the surroundings. Biodivers. Conserv. 19:837-852.

Systat Software Inc. 2006. SigmaStat for windows (version 3.5). 
Ulyshen, M.D. \& Hanula, J.L. 2009. Habitat associations of saproxylic beetles in the southeastern United States: A comparison of forest types, tree species and wood postures. Forest Ecol. Manag. 257:653-664.

Underwood, E.C., Viers, J.H., Klausmeyer, K.R., Cox, R.L. \& Shaw, M.R. 2009. Threats and biodiversity in the mediterranean biome. Divers. Distrib. 15:188-197. van Geffen, K.G., Poorter, L., Sass-Klaassen, U., van Logtestijn, R.S.P. \& Cornelissen, J.H.C. 2010. The trait contribution to wood decomposition rates of 15 Neotropical tree species. Ecology 91:3686-3697.

Villagrán, C. 1995. Quaternary history of the Mediterranean vegetation of Chile. En: Arroyo, M.T.K., Zedler, P.H. \& Fox, M.D. (eds.) Ecology and biogeography of Mediterranean ecosystems in Chile, California and Australia. Springer Verlag, Berlin. pp 3-20. 